\title{
SPACE GROUND INTERFEROMETER
}

\section{A. I. SAVIN, M.B. ZAXON \\ Corporation "Kometa" Moscow, USSR}

L. I. MATVEYENKO

Space Research Institute, Profsoyuznaya 84/32, Moscow 117810, USSR

ABSTRACT A space project for studying ecological Earth problems is being carried out by means of radio techniques. A $30 \mathrm{~m}$ prototype antenna has already been deployed and tested. The radio telescope will be launched in 1994 into a circular orbit haying an altitude $600 \mathrm{~km}$ and an inclination of 65 . The planned mission time is $\geq 1.5$ year; $25 \%$ of this will be available for VLBI observations at wavelengths 6 and $18 \mathrm{~cm}$.

Studies of the fine structure of quasars and radio galaxies have shown that clouds of relativistic plasma are injected from their nuclei and form "jets". The observed velocity of these clouds appears to exceed the velocity of light. Their brightness temperature $\mathrm{T}$ attains $10^{12} \mathrm{~K}$, the value determined by scattering by the inverse Compton effect (Kellermann and Paul iny-Toth 1969). Star formation processes in region of gas and dust are accompanied by maser emission and protostars are surrounded by protoplanet disks and rings. The brightness temperature of compact $\mathrm{OH}$ masers are typically $\mathrm{T}^{2} 10^{12} \mathrm{~K}$, for $\mathrm{H}_{2} \mathrm{O}$ masers they are $10^{14} \mathrm{~K}$, but for the "megamaser" "2 in Orion KL brightness temperature reaches $10^{17} \mathrm{~K}$ (Mat veyenko 1981).

For further studies of the structure and evolution of compact radio sources a higher angular resolution and a larger dynamic range are required; the latter implies a better coverage of the U,V plane

(Kogan 1972; Kostenko and Mat veyenko 1982; Sagdeev 1984 Kardashev and Slysh 1987). The angular resolution of the Space-Ground interferometer is limited by sensitivity and by scattering of the 
emission by the interstellar medium. The maximum baseline as determined by the sensitivity is

$$
B[m] \leq \frac{\pi}{4 n}\left[\frac{T_{b} \gamma d_{1} d_{2}\left[K_{1} K_{2}\right]^{0.8}(\Delta f \tau)^{0.8}}{b N\left(T_{1} T_{2}\right]^{0.8}}\right]^{0.0}
$$

where $d_{1}, d_{2}, K_{1}, K_{2}$ are diameters and efficiencies of the antennas, $T_{b}$ is the brightness temperature of the source, $T_{1}, T_{2}$ are the system noise temperature at the telescopes, $\Delta f$ is the bandwidth, $\tau$ is the coherent integration time, $b$ is the "b-factor" and $n=\phi / \theta$ is the ratio of the angular size of the source to the fringe spacing. For $n=0.5$, visibility $\gamma=0.5,\left(K_{1} K_{2}\right)^{0.8}=0.5$ and a signal to noise ratio $\mathrm{N}$ equal 10

$$
B[m] \leq 0.04\left[\frac{T_{b} d_{1} d_{2}[\Delta f \tau]^{0.8}}{\left[T_{1} T_{2}\right]^{0.5}}\right]^{0.0}
$$

The scattering angle is given by

$$
\theta_{\text {scat }}=10^{-2} \lambda^{2}|\sin b|^{-0.0}
$$

where $\lambda$ is the wavelength in meters and $b$ is galactic latitude. The corresponding baseline would be

$$
B[m] \leq 10^{7} \lambda^{-1}|\sin b|^{0.0}
$$

In Soviet Union a space project for studying ecological Earth problems is being carried out by means of radio techniques. A space radio telescope, $30 \mathrm{~m}$ in diameter, will be used: a full scale prototype antenna has already been deployed and tested. The antenna surface consists of stainless steel filaments $50 \mu \mathrm{m}$ in diameter with RMS deviation from a paraboloid smaller than a few mm. Its pointing would be accurate to $\leq 1$ arcmin. For space-ground interferometry the antenna would be 
used at wavelengths of 6 and $18 \mathrm{~cm}$ with system noise temperatures of 100 and $60 \mathrm{~K}$ respectively.

The expected antenna efficiency is 0.5 ; the bandwidth for transmitting data to the ground would be either 2 or $16 \mathrm{MHz}$. A rubidium clock, locked to a high quality crystal oscillator would provide the on board frequency and time standard; the resulting coherence time of 50-100 sec would be limited by the source structure. The radio telescope will be launched in 1994 by the "Proton" vehicle into a circular orbit haying an altitude of $600 \mathrm{~km}$ and an inclination of 65 . The planned mission time is $\geq 1$ years and $25 \%$ of this will be available for VLBI observations. The angular resolution of the Space-ground interferometer will be about 1 mas. The synthes is of the aperture with a diameter of about $13000 \mathrm{~km}$ will require about 12 hours. The sensitivity of the space antenna with a $70 \mathrm{~m}$ ground antenna is about $15-20 \mathrm{mJy}$ for $16 \mathrm{MHz}$ data bandwidth and $2 \mathrm{Jy}$ for $1 \mathrm{kHz}$ bandwidth (spectral line studies). The sensitivity is sufficient to study compact continuum sources with $\mathrm{T}_{\mathrm{b}} \geq 10^{10} \mathrm{~K}$; for the same sensitivity and $T_{b}=10^{12} \mathrm{~K}$, a baseline of $5.10^{4} \mathrm{~km}$ corresponding to the scattering 1 imit at $18 \mathrm{~cm}$ could be used. The scattering of $\mathrm{OH}$ sources located in the galactic plane limits the useful baselines to a few thousand $\mathrm{km}$.

\section{REFERENCES}

Kardashev, N.S. and Slysh V.I. 1987

Proc. I AU' Symposium No 129, Cambridge, 433.

Kellermann, K. I. and Pauliny-Toth, I. I.K. 1969, Ap. J., 155, L31.

Kogan, L. R. 1972, Ph. Doctor Degree Thesis, Moscow, IKI.

Kostenko, V.I., and Matveyenko, L. I. 1977, Preprint I KI No 340.

Mat veyenko, L. I. 1981, Sov. Let. Astron. J. 7, 100. Sagdeev, R.S. 1984, Proceedings of Workshop Held at Gross Enzersdorf, Austria, 19. 